\title{
Computationally Efficient Multi-Antenna Techniques for Multi-User Two-Way Wireless Relay Networks
}

\author{
Samer Alabed \\ Department of Electrical Engineering, American University of the Middle East, Kuwait
}

\section{Article Info}

Article history:

Received Sep 24, 2017

Revised Feb 8, 2018

Accepted Mar 16, 2018

\section{Keyword:}

MIMO systems

Multi-user two-way wireless relay networks

Multi-antenna techniques

Network coding

Cooperative diversity

Minimum mean squared error

Maximum likelihood (ML)

\begin{abstract}
In this work, we are interested in implementing, developing and evaluating multi-antenna techniques used for multi-user two-way wireless relay networks that provide a good tradeoff between the computational complexity and performance in terms of symbol error rate and achievable data rate. In particular, a variety of newly multi-antenna techniques is proposed and studied. Some techniques based on orthogonal projection enjoy low computational complexity. However, the performance penalty associated with them is high. Other techniques based on maximum likelihood strategy enjoy high performance, however, they suffer from very high computational complexity. The Other techniques based on randomization strategy provide a good trade-off between the computational complexity and performance where they enjoy low computational complexity with almost the same performance as compared to the techniques based on maximum likelihood strategy.
\end{abstract}

Copyright (c) 2018 Institute of Advanced Engineering and Science. All rights reserved.

\section{Corresponding Author:}

Name: Samer Alabed

Affiliation: Assistant professor

Address: Department of Electrical Engineering, American University of the Middle East, Block 3, Building 1, Egaila, Kuwait.

Phone: +965 22251400 Ext.: 1790

Email: Samer.Al-Abed@aum.edu.kw

\section{INTRODUCTION}

In a wireless network, relay station advantages can be evaluated through two parameters: performance and cost. From the performance side, relay stations can be utilized to extend the achivable data rate within the same cell or alternatively, they can be used to extend the coverage area [1]. When a relay station is installed to extend the coverage area, both, relay station and base station, use the same frequency at the same time which increases the spectrum reuse. Thus, the use of relay station improves the overall system throughput. By installing more base stations instead of relay stations, the same or in fact better performance can be achieved. However, installing base stations is much more expensive than installing relay stations.

Relay technology can be used in rural scenarios to extend the coverage [2]. It can be used in the case of earthquake or disasters where deploying a fixed line backhaul link for a base station is difficult. In the last decade, cooperative diversity strategies using randomly distributed relay nodes between the communicating terminals have been extensively studied as their improvements in performance do not require additional power or frequency spectrum [3-14]. The main objective of this work is to propose efficient relaying techniques to increase the sum rate and reduce the symbol error rate (SER) with low computational complexity.

\section{SYSTEM MODEL}

Let us consider a half duplex system which consists of $M$ single-antenna mobile stations (MSs) communicating with another $M$ single-antenna mobile stations via a relay station (RS) having $N(N \geq 2 M)$ antennas as shown in Fig. 1. There is no direct link between mobile stations and their communication partners. Relay station uses either the decode-and-forward (DF) or the amplify-and-forward (AF) protocol depending on the used technique. The noise at the relay station and at MS nodes is assumed to be modeled as independent, zero-mean, 
complex Gaussian random variable with variance $\sigma_{R S}^{2}$ and $\sigma^{2}$, respectively. Let us assume that the uplink and downlink channels are reciprocal and frequency flat fading. Further, the channels are assumed to remain constant during each transmission cycle. The maximum transmission power at the relay station and at the $i^{\text {th }} \mathrm{MS}$, i.e., $\mathrm{MS}_{i}$, is given by $P_{R S}$ and $P_{i}$, respectively. Furthermore, it is assumed that the channel state information (CSI) is available at the relay and mobile stations.

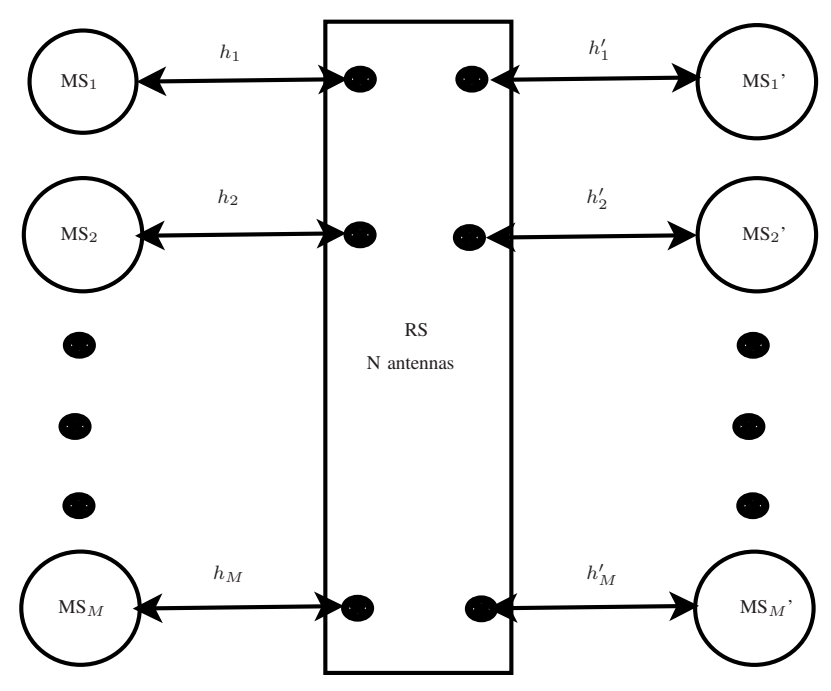

Figure 1. System model.

The combined multiple access channel $\mathbf{H} \in \mathbb{C}^{N \times 2 M}$ from all MSs to RS is given by

$$
\mathbf{H}=\left[\begin{array}{llllllll}
\mathbf{h}_{1} & \mathbf{h}_{2} & \ldots & \mathbf{h}_{M} & \mathbf{h}_{1}^{\prime} & \mathbf{h}_{2}^{\prime} & \ldots & \mathbf{h}_{M}^{\prime}
\end{array}\right]
$$

where $\mathbf{h}_{i}$ and $\mathbf{h}_{i}^{\prime}, i=1, \ldots, M$ are column vectors representing channel from $\mathrm{MS}_{i}$ to RS and from its corresponding partner, i.e., $\mathrm{MS}_{i}^{\prime}$, to RS, respectively. Similarly, broadcast channel from relay station to all MS nodes is given by $\mathbf{H}^{H} \in \mathbb{C}^{2 M \times N}$. For the given system model, in the first time slot, all users transmit their data to the relay station. The signal received at relay station $\mathbf{y}_{R} \in \mathbb{C}^{N \times 1}$ is given by

$$
\mathbf{y}_{R}=\sum_{i=1}^{M} \mathbf{h}_{i} s_{i}+\sum_{i=1}^{M} \mathbf{h}_{i}^{\prime} s_{i}^{\prime}+\mathbf{n}_{R}
$$

where $s_{i}$ and $s_{i}^{\prime}$ are the signals transmitted from the $\mathrm{MS}_{i}$ and $\mathrm{MS}_{i}^{\prime}$ nodes, respectively, to the relay station and $\mathbf{n}_{R}$ is the noise vector at the relay station in the first time slot. This signal needs to be processed in order to mitigate interference and noise. Let $\mathbf{G} \in \mathbb{C}^{N \times N}$ be the processing matrix at the relay station. In case of using the $\mathrm{AF}$ protocol, relay processing matrix is represented by a single matrix, i.e., $\mathbf{G}$ [15]. Whereas, in case of using the DF protocol, relay processing matrix is represented by a multiplication of three matrices $\mathbf{G}_{m}, \mathbf{G}_{b}$, and $\mathbf{W}$, such that

$$
\mathbf{G}=\mathbf{G}_{m} \mathbf{W G}_{b}
$$

where $\mathbf{G}_{m}$ is used to remove the effect of the interference occurring in the multiple access phase, i.e. during the first time slot, permutation matrix $\mathbf{W}$ is then used to rearrange the resulting vector in a proper order before sending it, and $\mathbf{G}_{b}$ is used to remove the effect of the interference occurring in the broadcast phase, i.e., during the second time slot. After processing the received signal $\mathbf{y}_{R}$ defined in (2) at the relay by using relay processing matrix $\mathbf{G}$, the signal vector $\mathbf{x}_{R}$ is obtained which is then transmitted to all mobile stations in the second time slot.

\section{TECHNIQUES TO MITIGATE INTERFERENCE}

This section presents proposed relaying techniques to mitigate interference. The orthogonal projection technique enjoys low computational complexity, however it suffers from low performance in terms of SER [15]. The other technique is based on maximum likelihood (ML) strategy to detect the symbol vector and then uses minimum mean square error (MMSE) strategy to broadcast the resulting vector. This technique enjoys optimal 
performance in terms of SER, however it suffers from high decoding complexity due to the use of ML detector at the relay. More techniques are also proposed in this section in order to reduce the overall computational complexity and improve the overall system performance using network coding and randomization strategy as explained in the next subsections.

\subsection{Multi-antenna technique based on ML and MMSE strategy}

In the technique proposed in [15], a zero-forcing strategy is used to reduce the effect of the interference at the cost of noise enhancement [16]. In order to improve the previous technique, other strategies can be used to reduce the effects of interference. Note that both, the interference in the multiple access phase and in the broadcast phase, need to be mitigated. In this technique, ML detector explained in [17] is used to detect the received signals at the relay during the first time slot. To mitigate the interference occurring in the broadcast phase, MMSE strategy is applied.

\subsubsection{ML detector}

As explained in Sec. 2., we are considering a multi-user system where all users are transmitting their signals at the same time to the relay station and the received signal vector at the relay station $\mathbf{y}_{R}$ is given by (2). The key idea of ML detector is to find the joint error $\mu$ for each possible combination of the transmit symbols, such as

$$
\mu=\sum_{n=1}^{N}\left|\mathbf{y}_{R}(n)-\sum_{i=1}^{M}\left(\mathbf{h}_{i}(n) s_{i}+\mathbf{h}_{i}^{\prime}(n) s_{i}^{\prime}\right)\right|^{2}
$$

where $\mathbf{y}_{R}(n)$ is the $n$th element of the vector $\mathbf{y}_{R}$. After calculating the value of $\mu$ for each possible combination of the transmit symbols from all mobile stations, the detected transmit symbol vector $\hat{\mathbf{s}} \in \mathbb{C}^{2 M \times 1}$ which gives minimum value of $\mu$ is obtained. ML provides an optimal solution to the detection problem, however it suffers from extremely high decoding complexity due to the exhaustive search over all possible combinations of symbols where its decoding complexity increases exponentially with the increase of the constellation size and the number of transmitted symbols.

\subsubsection{Permutation matrix}

After detecting the symbols optimally using ML detector, the symbols need to be arranged in a vector in a proper sequence in order to receive them correctly at the destination node. The permutation matrix $\mathbf{W} \in \mathbb{C}^{2 M \times 2 M}$ used in (3) is given by

$$
\mathbf{W}=\left(\begin{array}{cc}
\mathbf{0}_{M \times M} & \mathbf{I}_{M} \\
\mathbf{I}_{M} & \mathbf{0}_{M \times M}
\end{array}\right)
$$

where $\mathbf{0}_{M \times M}$ and $\mathbf{I}_{M \times M}$ denote an $M \times M$ matrix which contains zeros in all its entries and an $M \times M$ identity matrix, respectively. The detected symbol vector $\hat{\mathbf{s}}$ is multiplied by the permutation matrix $\mathbf{W}$, such that

$$
\mathbf{t}=\mathbf{W} \hat{\mathbf{s}}
$$

\subsubsection{MMSE strategy}

To mitigate the interference in the broadcast phase, MMSE filter is used. This filter is represented by the matrix $\mathbf{G}_{b}$ used in (3). After normalization, it is given by

$$
\mathbf{G}_{b}=\alpha \tilde{\mathbf{G}}_{b}
$$

where $\tilde{\mathbf{G}}_{b}$ is the MMSE filter, given by

$$
\tilde{\mathbf{G}}_{b}=\mathbf{H}\left(\mathbf{H}^{H} \mathbf{H}+1 / \gamma \mathbf{I}_{N}\right)^{-1}
$$

and $\alpha$ is the factor to fulfill power constraint at the relay station, given by

$$
\alpha=\frac{\sqrt{\mathbf{P}_{R S}}}{\sqrt{\left\|\tilde{\mathbf{G}}_{b}\right\|^{2}}} .
$$


The transmitted signal vector from relay station can then be obtained as

$$
\mathbf{x}_{R}=\mathbf{G}_{b} \mathbf{t}
$$

The signal given by (10) is then received by all mobile stations where the signal received at the $i^{\text {th }}$ mobile station $\mathrm{MS}_{i}$ is given by

$$
y_{i}=\mathbf{h}_{i}^{H} \mathbf{x}_{R}+n_{i}
$$

In this technique, $\mathrm{MS}_{i}$ needs only its CSI, i.e., $\mathbf{h}_{i}$. The achievable sum rate of this system can be calculated by using

$$
R_{\text {sum }}=\frac{1}{2} \sum_{i=1}^{2 \times M} \log _{2}\left(1+\gamma_{i}\right)
$$

and the receive signal to interference and noise ratio (SINR) at the $\mathrm{MS}_{i}$ in this case is given by

$$
\gamma_{i}=\frac{\left|\mathbf{h}_{i}^{H} \mathbf{G}_{b i}\right|^{2}}{\sigma^{2}+\sum_{j \neq i}\left|\mathbf{h}_{i}^{H} \mathbf{G}_{b j}\right|^{2}}
$$

where $\mathbf{G}_{b i}$ represents the $i^{t h}$ column of the relay transmit filter $\mathbf{G}_{b}$.

\subsection{Multi-antenna technique based on MMSE strategy}

In this technique, MMSE filter is applied at the relay twice. First, as a receive filter at the relay during the first time slot. Second, as a transmit filter before sending the decoded data from the relay station in the second time slot. During the first time slot, the MMSE detector at the relay is given by

$$
\mathbf{G}_{m}=\left(\mathbf{H}^{H} \mathbf{H}+1 / \gamma \mathbf{I}_{N}\right)^{-1} \mathbf{H}^{H} .
$$

Using this detector, the estimated signal vector $\hat{\mathbf{s}}$ is obtained as

$$
\hat{\mathbf{s}}=\mathbf{G}_{m} \mathbf{y}_{R}
$$

This estimated vector is then hard decoded and used for further processing. Note that the estimated vector without hard decoding could also be used. The decoded symbols need to be rearranged to make sure that every node receives symbol from its corresponding partner. This can be achieved by using permutation matrix given by (5). The rearranged vector $\mathbf{t}$ is given by (6). Before transmitting this signal vector, transmit MMSE filter given by (7) is performed. Sec. 3.1.3. also explains the calculation of the power normalization factor $\alpha$ expressed in (9) for this filter. The final transmit vector $\mathbf{x}_{R}$ is given by

$$
\mathbf{x}_{R}=\mathbf{G} y_{R}
$$

where $\mathbf{G}$ is defined in (3). The achievable sum rate using this technique can be calculated using (12).

\subsection{Multi-antenna technique based on network coding}

\subsubsection{Concept}

Network coding can be useful in combining signals to transmit them using less number of time slots [3, 7-12]. In multi-antenna scenarios, network coding combines signals to reduce the number of transmitted symbols which can then be transmitted over less number of antennas. Otherwise, if the same number of antennas is used to transmit these combined symbols, a better performance can be achieved. In this technique, M-PSK modulation scheme is used. Making use of the fact that when two M-PSK symbols lying on the unit circle are multiplied, the resultant symbol lies on the same circle. Because of this property, $\mathrm{E}\left\{\mathbf{s s}^{H}\right\}$ is preserved even after multiplication is performed.

\subsubsection{Implementation}

As explained earlier, the basic idea behind this technique is to use network coding at the relay to improve the performance in terms of sum rate as well as SER. The received signal vector at the relay station is given by (2). This received signal is the sum of all the signals coming from all the mobile stations. A receive filter is needed at the relay station to separate the signals and thus detect the correct signals from all the nodes. In this technique, MMSE filter given by (14) is applied. The estimated signal vector $\hat{\mathbf{s}}$ is obtained using (15). The symbol vector $\hat{\mathbf{s}}$ 
is then hard decoded based on the decision boundary of the used modulation scheme. Note that the signal vector $\hat{\mathbf{s}}$ can also be sent without hard decoding. In the next step, symbols belonging to the same communication pair are multiplied to obtain a new combined symbol. The new symbol $t_{k}$, generated from the symbols of the $k^{t h}$ pair, is given by

$$
t_{k}=\hat{s}_{k} \cdot{\hat{s^{\prime}}}_{k} \quad k=1, \ldots, M
$$

To keep the symbol $t_{k}$ generated for the $k^{t h}$ pair separate from the symbols of the other pairs, the orthogonal projection strategy is applied. The key idea of the orthogonal projection strategy is to find a precoding matrix at the relay that groups the signals from the same pair together and eliminates the inter-pair interference [15]. The constraint for finding such a precoding matrix is $N \geq 2 M-1$ which is fulfilled by our system model. In this strategy, $\mathrm{MS}_{i}$ needs to know the relay precoding matrix in order to remove the effect of self-interference which means that the CSI is required at the mobile stations. The precoding matrix for the $k^{t h}$ communicating pair is given by

$$
\mathbf{P}_{k}=\left[\mathbf{h}_{1} \ldots \mathbf{h}_{k-1} \mathbf{h}_{k+1} \ldots \mathbf{h}_{M} \mathbf{h}_{1}^{\prime} \ldots \mathbf{h}_{k-1}^{\prime} \mathbf{h}_{k+1}^{\prime} \ldots \mathbf{h}_{M}^{\prime}\right]
$$

where $\mathbf{P}_{k} \in \mathbb{C}^{N \times(2 M-2)}$ is a submatrix of channel matrix $\mathbf{H}$, given by (1). $\mathbf{P}_{k}$ is obtained by removing the $k^{t h}$ and $(k+M)^{t h}$ columns from $\mathbf{H}$. The orthogonal projection matrix $\mathbf{Q}_{k} \in \mathbb{C}^{N \times N}$ is obtained from interference channel $\mathbf{P}_{k}$ and is given by

$$
\mathbf{Q}_{k}=\left(\mathbf{I}_{N}-\mathbf{P}_{k}\left(\mathbf{P}_{k}^{H} \mathbf{P}_{k}\right)^{-1} \mathbf{P}_{k}^{H}\right) \quad k=1, \ldots, M .
$$

$\mathbf{Q}_{k}$ is then multiplied by the received signal $\mathbf{y}_{R}$ given by (2) to recover the signals of the $k^{t h}$ pair, such as

$$
\mathbf{Q}_{k} \mathbf{y}_{R}=\mathbf{Q}_{k} \mathbf{h}_{k} s_{k}+\mathbf{Q}_{k} \mathbf{h}_{k}^{\prime} s_{k}^{\prime}+\mathbf{Q}_{k} \mathbf{n}_{R}
$$

Note that $\mathbf{Q}_{k} \mathbf{h}_{i}=0$ and $\mathbf{Q}_{k} \mathbf{h}_{i}^{\prime}=0(k \neq i)$. Let us define a matrix $\mathbf{1} \in \mathbb{C}^{N \times 1}$ which contains ones in all its entries. The transmitted signal from the relay is given by

$$
\mathbf{x}_{R}=\alpha \sum_{k=1}^{M} \mathbf{Q}_{k} \cdot \mathbf{1} \cdot \hat{t}_{k}
$$

where $\alpha$, used to normalize the transmit signal power at the relay station in order to fulfill the relay station power constraint, is given by

$$
\alpha=\frac{\sqrt{\mathbf{P}_{R S}}}{\sqrt{\sum_{k=1}^{M}\left\|\mathbf{Q}_{k}\right\|^{2}}} .
$$

The received signal at the $i^{t h}$ node $\mathrm{MS}_{i}$ during the second time slot is given by

$$
y_{i}=\mathbf{h}_{i}^{H} \mathbf{x}_{R}+n_{i}
$$

In this technique, $\mathrm{MS}_{i}$ needs only its CSI, i.e., $\mathbf{h}_{i}$. The achievable sum rate of the system can be calculated using (12) where SINR at the node $\mathrm{MS}_{k}$ is given by

$$
\gamma_{k}=\frac{\left|\sum_{j=1}^{N} \alpha \mathbf{h}_{k}^{H} \mathbf{Q}_{k j}\right|^{2}}{\sigma^{2}}
$$

and $\mathbf{Q}_{k j}$ is the $j^{t h}$ column of the relay precoding matrix $\mathbf{Q}_{k}$ for the $k^{t h}$ pair nodes, i.e., $\mathbf{M S}{ }_{k}$ and $\mathrm{MS}_{k}$.

\subsection{Randomization techniques}

\subsubsection{Concept}

In the techniques explained in Sec. 3.2. and Sec. 3.3., the MMSE strategy is performed at the relay to mitigate the interference occurring in the first time slot using (14). The techniques based on MMSE strategy with or without network coding are simple and enjoy a low decoding complexity, however, they suffer from low performance in terms of SER as compared to the techniques based on ML strategy. To improve the performance of the techniques based on MMSE strategy during the first time slot, let us search randomly for a symbol vector having same mean and variance as our estimated MMSE symbol vector defined in (15). In other words, the randomization strategy is used during the first time slot to find a better estimated symbol vector at the relay and thus to reduce the overall error rate. 
The signal received at the relay $\mathbf{y}_{R}$ is given by (2) and the MMSE filter $\mathbf{G}_{m}$ used for obtaining the estimated symbol vector $\hat{\mathbf{s}}$ is given by (14). This estimated vector $\hat{\mathbf{s}}$ is obtained by (15). After receiving $\mathbf{y}_{R}$ and obtaining $\hat{\mathbf{s}}$ using MMSE filter, a new random symbol vector $\hat{\mathbf{s}}_{n e w}$ is generated with mean and variance equal to the original estimated symbol vector $\hat{\mathbf{s}}$. Afterwards, two error values for the two estimated symbol vectors, $\hat{\mathbf{s}}$ and $\hat{\mathbf{s}}_{\text {new }}$, are generated using

$$
\begin{aligned}
e & =\left\|\mathbf{y}_{R}-\mathbf{H} \hat{\mathbf{s}}\right\|^{2}, \\
e_{\text {new }} & =\left\|\mathbf{y}_{R}-\mathbf{H} \hat{\mathbf{s}}_{\text {new }}\right\|^{2} .
\end{aligned}
$$

If $e_{n e w}<e$ is true, this means that we have found a better estimated symbol vector. Therefore, the original estimated symbol vector $\hat{\mathbf{s}}$ can then be discarded and the new one, i.e., $\hat{\mathbf{s}}_{n e w}$, can be stored instead. If the original estimated symbol vector $\hat{\mathbf{s}}$ is better, then the newly obtained one, i.e., $\hat{\mathbf{s}}_{n e w}$ is discarded. We again try to find an estimated symbol vector better than the obtained one in the last step. For that, we again follow the same procedure of finding a new random symbol vector, comparing it with the existing one, and then storing the best one. This process needs to be repeated for a pre-defined number of iterations to obtain a better estimated symbol vector. The randomization technique based on MMSE without using network coding is explained above and named as the randomization technique based on MMSE strategy. This strategy, i.e., randomization strategy, can be combined also with the techniques based on network coding explained in Sec. 3.3. in order to improve the estimated symbol vectors of each pair before combining them using (17). The latter technique is named as the randomization technique based on network coding.

\section{RESULTS AND DISCUSSION}

This section presents the simulation settings and the results obtained for the techniques described in Sec. 3 .. The performance of the proposed techniques and the one proposed in [15] are compared using two parameters: average achievable sum rate and symbol error rate. A Rayleigh flat fading reciprocal channel is assumed for uplink and downlink communication. Channel vectors are assumed to be independent and identical distributed (i.i.d) and remain constant during the whole transmission cycle. The whole CSI is assumed to be available at the relay station while mobile stations require their own CSI as explained in Sec. 3.. For all simulations, the number of mobile station pairs intending to communicate with each other is set to $M=2$ and number of antennas at the relay station are set to $N=4$. Transmit signal to noise ratio (SNR) is varied from $0 \mathrm{~dB}$ to $30 \mathrm{~dB}$. The power at each mobile station is $P_{i}=1, \quad \forall i$. The power at the relay is assumed to be proportional to the number of antennas at relay, thus, $P_{R}=N=4$. The noise power is assumed to be changing in accordance with the transmit SNR requirement.

\subsection{Symbol Error Rate}

To find the SER at each SNR value, 500000 symbols are transmitted from each MS. In the techniques based on randomization strategy, 30 iterations of randomization are used. Fig. 2 shows the performance of each technique discussed in Sec. 3. and the one proposed in [15] in terms of SER. In Fig. 2, the legend OP, MLtxMMSE, MMSEtxMMSE, MMSESigMulOP, MMSErandSigMulOP, and MMSErandtxMMSE denote the technique proposed in [15], the technique based on ML strategy explained in Sec. 3.1., the technique based on MMSE strategy explained in Sec. 3.2., the technique based on network coding explained in Sec. 3.3., the randomization technique based on network coding explained in Sec. 3.4., and the randomization technique based on MMSE strategy explained in Sec. 3.4., respectively. It is clearly visible that the proposed technique based on the optimal ML detector, denoted by MLtxMMSE, enjoys the best performance and outperforms the other techniques, however, as explained in Sec. 3.1., it suffers from extremely high decoding complexity. On the other hand, the proposed randomization technique based on MMSE, denoted by MMSErandtxMMSE, enjoys low decoding complexity with almost the same performance as compared to the one based on the optimal ML detector. Moreover, the randomization strategy can surely improve the performance drastically in a comparatively less complex way.

\subsection{Average achievable sum rate}

Fig. 3 shows the performance of the proposed techniques and the technique proposed in [15] in terms of achievable sum rate where the legend OP, MMSE, and SigMulOP denote the technique proposed in [15], the technique based on MMSE strategy which is explained in Sec. 3.1. and Sec. 3.2., and the technique based on network coding which is explained in Sec. 3.3., respectively. As explained in Sec. 3.3., the technique based on network coding reduces the number of transmitted symbols by combining them, therefore, Fig. 3 shows a significant gain in the sum rate achieved in the case of the technique based on network coding as compared to the other techniques. 


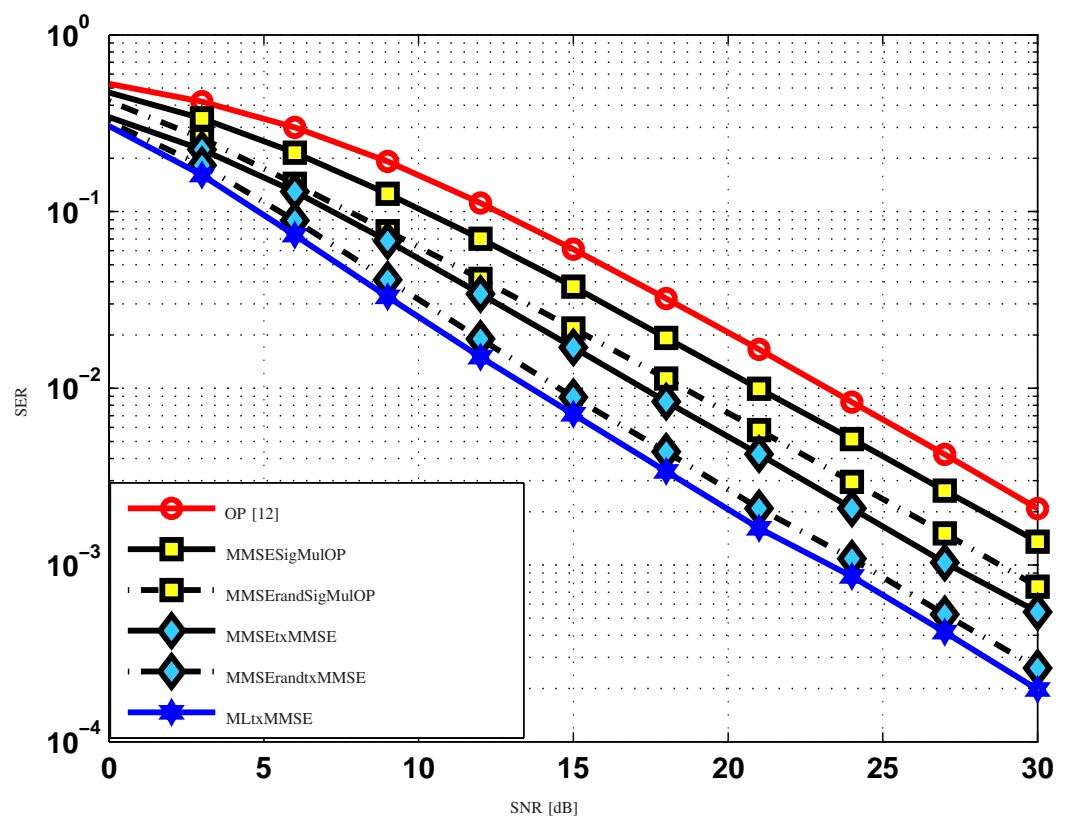

Figure 2. Symbol error rate vs SNR (dB).

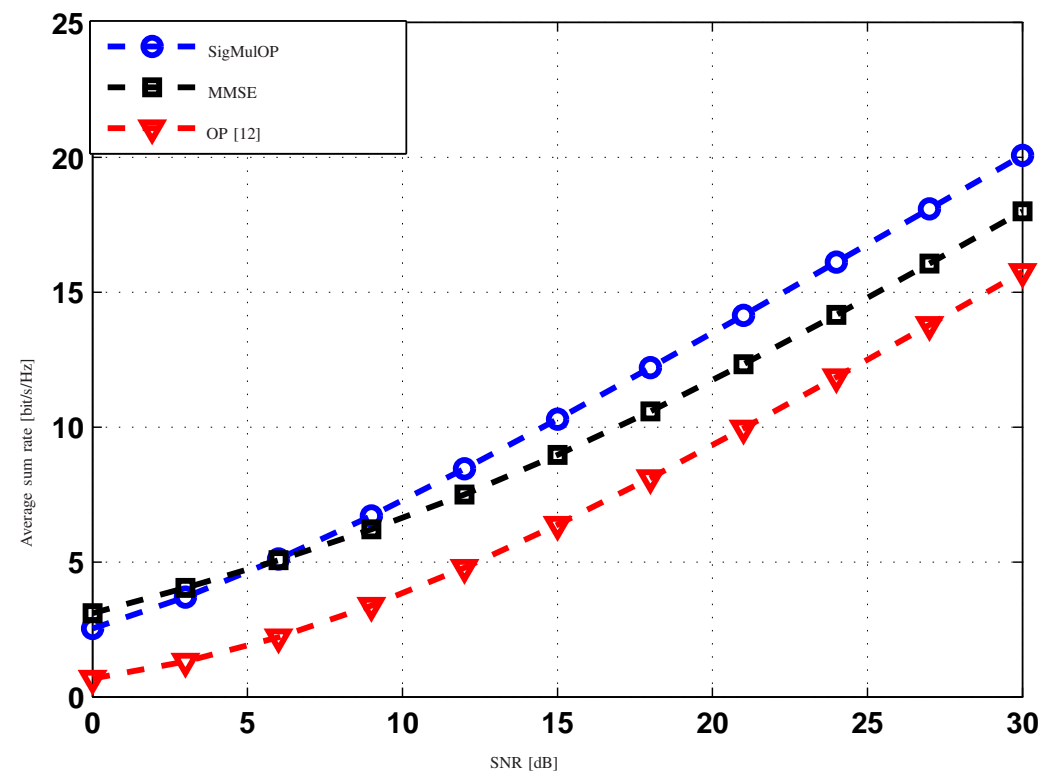

Figure 3. Average achievable sum rate vs SNR (dB). 


\section{CONCLUSION}

In this work, the performance in terms of SER and achievable data rate of a variety of newly multi-antenna techniques used for multi-user two-way wireless relay networks are proposed and studied. The multi-antenna techniques based on orthogonal projection enjoys low computational complexity. However, the performance penalty associated with them is high. Moreover, the techniques based on ML strategy enjoys high performance in terms of SER, however, they suffer from very high decoding complexity. On the other hand, the proposed randomization technique based on MMSE strategy enjoys low decoding complexity with almost the same performance as compared to the technique based on ML strategy.

\section{REFERENCES}

[1] R. Pabst, B. H.Walke, D. Schultz, P. Herhold, H. Yanikomeroglu, S. Mukherjee, H. Viswanathan, M. Lott, W. Zirwas, M. Dohler, H. Aghvami, D. Falconer, and G. Fettweis, "Relay-based deployment concepts for wireless and mobile broadband radio," IEEE Communications Magazine, vol. 42, no. 9, pp. 80-89, 2004.

[2] M. Iwamura, H. Takahashi, and S. Nagata, "Relay technology in LTE-Advanced," NTT DoCoMo Technical Journal, vol. 12, no. 2: pp. 29-36, 2010.

[3] S. Alabed and M. Pesavento, "Distributed differential space-time coding techniques for two-way wireless relay networks," the 16th International IEEE/ITG Workshop on Smart Antennas (WSA 2012), Dresden, Germany, November 2011.

[4] Nasaruddin, Yunida, Khairul Munadi, "Improved model of the selection with soft and hard combining decoding strategies for multi-user multi-relay cooperative networks," International Journal of Electrical and Computer Engineering (IJECE), vol. 6, no. 4, pp. 1766 1778, August 2016.

[5] C. Preetham, M. Prasad, D. Saranya, C. Somepalli, D. Krishna, and V. Rohit, "Performance Analysis of Cooperative Hybrid Cognitive Radio Network with Various Diversity Techniques," International Journal of Electrical and Computer Engineering (IJECE), vol. 6, no. 5, pp. 2125 2133, October 2016.

[6] Nasaruddin, et al., "Optimized power allocation for cooperative amplify-and-forward with convolutional codes," TELKOMNIKA Indonesian Journal of Electrical Engineering, vol. 12, no. 8, pp. 6243-6253, 2014.

[7] S. Alabed, M. Pesavento, and A. Klein, "Relay selection based space-time coding for two-way wireless relay networks using digital network coding," In Proceedings of the Tenth International Symposium on Wireless Communication Systems, Ilmenau, TU Ilmenau, Germany, Aug. 27-30, 2013.

[8] S. Alabed, "Performance Analysis of Two-Way DF Relay Selection Techniques," Elsevier ICT Express, DOI: 10.1016/j.icte.2016.08.008, September 2016.

[9] S. Alabed, J. Paredes, and A. B. Gershman, "A simple distributed space-time coded strategy for two-way relay channels," IEEE Transactions on Wireless Communications, pp. 1260-1265, vol. 11, no. 4, April, 2012.

[10] S. Alabed, M. Pesavento, and A. Gershman, "Distributed differential space-time coding techniques for twoway wireless relay networks," In Proceedings of the Fourth IEEE International Workshop on Computational Advances in Multi-Sensor Adaptive Processing (CAMSAP 11), pp. 221-224, San Juan, Puerto Rico, December 2011.

[11] S. Alabed, M. Pesavento, and A. Klein, "Distributed differential space-time coding for two-way relay networks using analog network coding," In Proceedings of the 1st European Signal Processing Conference (EUSIPCO’13), Marrakech, Morocco, Sep. 9-13, 2013.

[12] S. Alabed, M. Pesavento, and A. Klein, "Non-coherent distributed space-time coding techniques for two-way wireless relay networks," EURASIP Special Issue on Sensor Array Processing, Feb. 2013.

[13] S. Alabed, J. Paredes, and A. Gershman, "A low complexity decoder for quasi-orthogonal space-time block codes," IEEE Transactions on Wireless Communications, vol. 10, no. 3, March 2011.

[14] A. Schad, S. Alabed, H. Degenhardt, and M. Pesavento, "Bi-directional differential beamforming for multiantenna relaying," 40th IEEE International Conference on Acoustics, Speech and Signal Processing, 2015.

[15] Z. Zhao, Z. Ding, M. Peng, W. Wang, and K. Leung, "A Special Case of Multi-Way Relay Channel: When Beamforming is not Applicable," IEEE Transactions on Wireless Communications, vol. 10, no. 7, pp. 20462051, 2011.

[16] H. Jafarkhani, "Space-Time Coding: Theory and Practice," Cambridge, U.K. Cambridge Univ. Press, 2005.

[17] B. Bjecke and J. Proakis, "Multiple transmit and receive antenna diversity techniques for wireless communications," Adaptive Systems for Signal Processing, Communications, and Control Symposium 2000, pp. 70-75, 2000 . 